\title{
Polymorphism of Merkel cells from slowly adapting type I mechanoreceptors in the buccal pouch of the golden hamster
}

\author{
Masakazu Tazaki ${ }^{1}$, Yukı Tazaki ${ }^{1}$, Takashi Inoue ${ }^{2}$ and Masakı Shimono ${ }^{2}$ \\ Oral Health Science Center, ${ }^{1}$ Department of Physiology and ${ }^{2}$ Department of Pathology, Tokyo Dental College, 1-2-2, Masago, \\ Mihamaku, Chiba 261-8502, Japan
}

(Received 1 March 2000; and accepted 24 March 2000)

\begin{abstract}
The shape of Merkel cells from Merkel cell-neurite complexes, which are slowly adapting type I (SAI) mechanoreceptors in the touch dome of 3-12 week old golden hamster cheek pouch mucosa, were examined by immunohistochemistry and under an electron microscope. This is the first report of its kind observing the shapes of Merkel cells which had beforehand been established functionally as SAI mechanoreceptors. Immunohistochemistry using anticytokeratin 20 antibody and light microscopic observations of quinacrine fluorescence treated Merkel domes revealed that some Merkel cells were of dendritic shape. On the other hand, many Merkel cells were of globular and/or roundish shape. Observation of Merkel cells using an electron microscope also revealed some dendritic-shaped Merkel cells, however almost all of them contacted nerve terminals. In spite of the Merkel cells shape, it seems appropriate to theorize that the majority of Merkel cells (including dendritic-shaped Merkel cells) from Merkel cell-neurite complexes in SAI mechanoreceptores are related to the reception of mechanical stimulation.
\end{abstract}

Immunohistochemical studies using various antibodies against Merkel cell specific cytokeratins have revealed a polymorphism of Merkel cells in the skin $(4,5)$ and mucosa $(6,7)$. Merkel cells in embryonic, fetal and adult human skin were reported to appear in two completely different forms: typical (globular and/or roundish) Merkel cells (RMCs) and dendritic Merkel cells (DMCs) possessing thread-like cell processes $(4,5)$. Using immunohistochemical and electron microscopic techniques, it has also been reported that the DMCs did not have contact with nerve terminals in the rodent palatine mucosa $(6,7)$. These

\footnotetext{
*Correspondence to: Dr Masakazu Tazaki Oral Health Science Center, Department of Physiology, Tokyo Dental College, 1-2-2, Masago, Mihamaku, Chiba 261-8502, Japan.

Tel.: +81 43270 3771; Fax: +81 43279 2052; E-mail:mtazaki@tdc.ac.jp
}

morphological findings for the polymorphism of Merkel cells suggested that there may be a heterogeneity of Merkel cells with regard to the functions of the cells. However, the above mentioned reports did not examine the function of Merkel cells.

On the other hand, Merkel cells in the touch dome of hamster cheek pouch mucosa were first reported by Calderon-Solt and Solt (1) using a transmission electron microscope. Tazaki and Iggo (9) reported that Merkel cell-neurite complexes of the touch dome were functioning as slowly adapting type I (SAI) mechanoreceptors. In the same preparation voltage dependent calcium channels (10) and stretch activated channels (11) were found in the Merkel cell membrane. Therefore, the function of the Merkel cells in the touch domes supported the hypothesis that the Merkel cells are the mechano-electric transducer in SAI receptors. The polymorphism and im- 
munohistochemistry of Merkel cells in the Merkel cell-neurite complexes functioning as SAI mechanoreceptors $(2,3,9)$ have thus so far not been carried out.

The cheek pouch mucosae of 3-12 week old golden male hamsters were used in this study. The touch domes were obtained under sodium pentobarbiturate anaesthesia (Nembutal; $50 \mathrm{mg} /$ $\mathrm{kg}$ i.p.). In order to mark Merkel cells with quinacrine fluorescence, hamsters were injected intreperitonealy with quinacrine dihydrochloride (Sigma; $15 \mathrm{mg} / \mathrm{kg}$ ) dissolved in distilled water at around 24 hours before the experiment. The fluorescent cells which are Merkel cells were immediately photographed using an Axiophot 2 microscope system (Zeiss) while the fluorescence faded rapidly under the UV light. For immunohistochemistry, the touch domes were fixed in $10 \%$ neutral formalin for $1 \mathrm{~h}$ at room temperature. The specimens were dehydrated through a graded ethanol series and embedded in paraffin. Paraffin prepared serial sections of $5 \mu \mathrm{m}$ in thickness were cut from the touch domes. After deparaffinization, sections were incubated in $0.05 \%$ trypsin for 30 minutes at room temperature. They were also incubated with either a monoclonal antibody against cytokeratine 20 (CK20) or a polyclonal rabbit antibody against proteingene product 9.5 (PGP 9.5) at $4^{\circ} \mathrm{C}$ overnight. The presence of the peroxidase was visualised resulting from a diaminobenzidine reaction. Finally, sections were counterstained in hematoxylin and coverslipped. For an electron microscopical observation, touch domes used for electropysiological recordings were fixed in a solution of the $1 \%$ glutaraldehyde and $1 \%$ formaldehyde in $0.1 \mathrm{M}$ sodium cacodylate buffer ( $\mathrm{pH}$ 7.3). Touch domes were then removed and postfixed in $1 \%$ osmium tetroxide in the same buffer for $2 \mathrm{~h}$ at $4^{\circ} \mathrm{C}$, dehydrated through a graded ethanol serics and cmbedded in Epon 812. After staining with uranyl acetate-lead citrate, they were examined under an electron microscope (H-600, HITACHI).

Using quinacrine-fluorescence and immunohistochemistry of anti-CK20, the distribution and shapes of Merkel cells in the touch domes of the hamster cheek pouch are shown in Fig. 1 A and B. The Merkel cells were only found under the hamster touch dome (Fig. 1A, bright cells) and located at the base of epithelial rete ridge in the touch domes (Fig. 1B, dark stained cells). Using immunohistochemistry of anti-PGP 9.5, the distribution of the nerve elements were also restricted to be around the basal cell layer of the epithelial rete ridge and groove in the lamina propria (not shown). These results also implied that Merkel cells in the hamster touch dome have contacts with the nerve terminals and are organized in Merkel cell-neurite complexes, as described in a previous report with methylene blue vital staining (8).

In recent immunohistochemical studies, it has been suggested that morphological findings of DMCs might be an indication of heterogeneity in Merkel cells possibly relating to different functions of the cells (7). Therefore, the shapes of Merkel cells in the hamster touch domes were observed using quinacrine-fluorescence and immunohistochemistry of anti-CK20 (Fig. 1A and B). The shapes of some Merkel cells in the touch domes were of dendritic shape as observed (Fig. $1 \mathrm{~A}$ and $\mathrm{B}$, arrows) in the palatine mucosa of adult rodents (7) and many Merkel cells were actually elliptical and/or roundish shaped. In the palatine mucosa of the hamster using immunohistochemistry of anti-CK20, many dendritic-shaped Merkel cells were also observed (not shown) and were more dendritically shaped than usual dendritic-shaped Merkel cells in the touch domes. The relative proportion of dendritic-shaped Merkel cells in the palatine mucosa was higher than that of dendritic-shaped Merkel cells in the touch domes although this study did not carry out a precise quantitative evaluation of dendriticshaped Merkel cells in the touch domes. It appears, that Merkel cells in the palatine mucosa of adult rodents including hamsters are highly polymorphic (7). The results of Merkel cells in Merkel cell-neurite complexes of the touch dome of golden hamsters differed from the results observed in the palatine mucosa, because many Merkel cells were elliptical and/or roundish shaped. Dendritic-shaped Merkel cells under the light microscopic observations in the hamster touch domes appeared as the DMCs as was reported in Tachibana et al. (7)

The Merkel cells and nerve terminals of the hamster touch domes were observed by electron microscopy, since the DMCs did not contact nerve terminals as described in Tachibana et al. (7). In our electron microscopical observations, many roundish-shaped Merkel cells and some dendritic-shaped Merkel cells were observed in the epithelial rete ridge of the touch domes in the hamster cheek pouch similar to the results observed using immunofluorescence microscopy. Fig. 

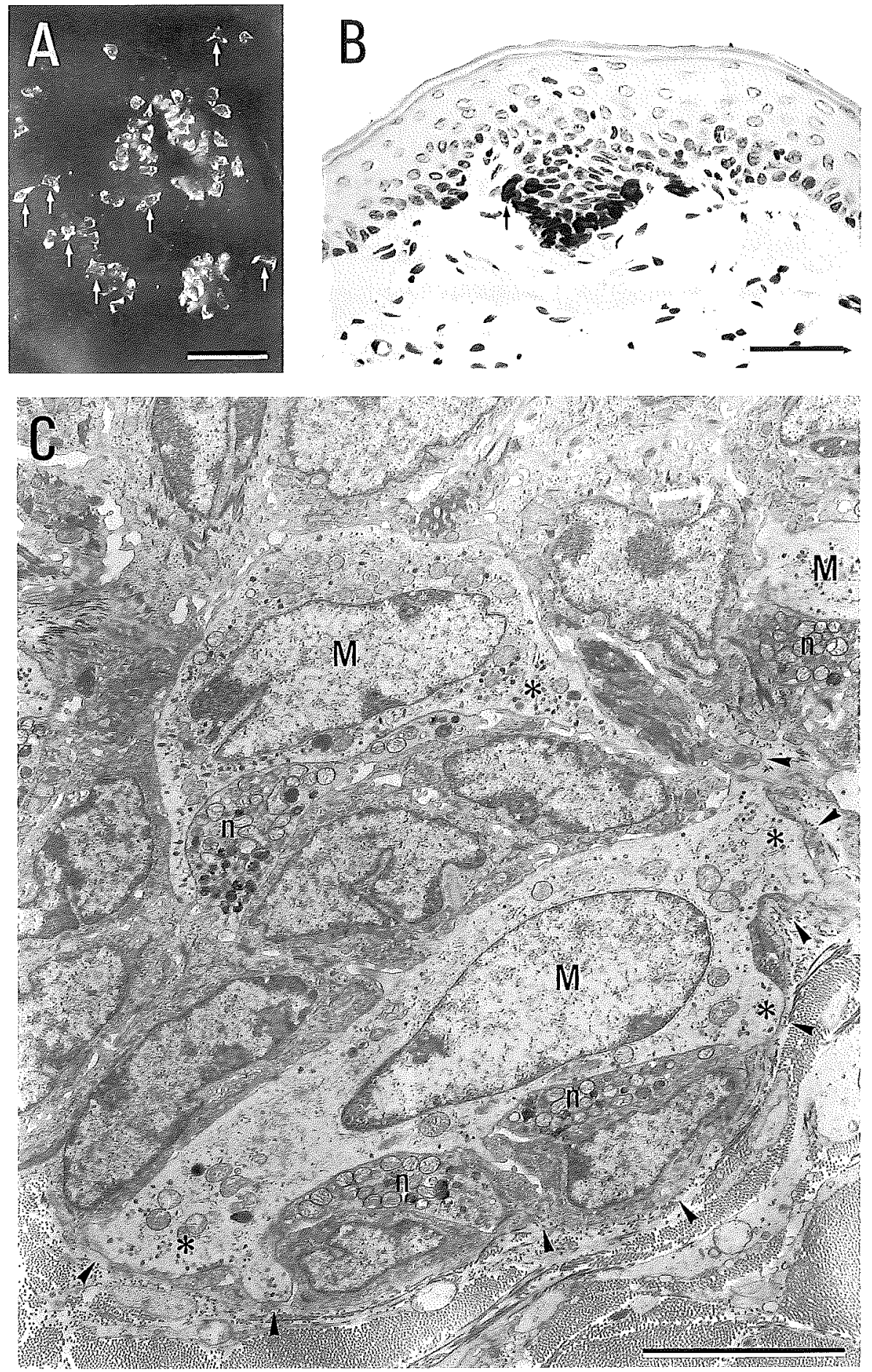

Fig. 1 A: Quinacrine-fluorescent Merkel cells in the touch dome of a hamster cheek pouch. Dendritic-shaped Merkel cells are shown by arrows. B: Merkel cells in the touch dome of the hamster were visualized by immunoperioxidase histochemistry using anti-CK20. Dendritic-shaped Merkel cells are shown by arrows. Scale bars $50 \mu \mathrm{m}$ in A and B. C: Electron micrograph of Merkel cells (M) in Merkel cell-neurite complexes of the touch dome of a hamster cheek pouch. Two dendritic-shaped Merkel cells possessing cytoplasmic process (asterisks) containing abundant neurosecretory granules are shown. They had contacts with the nerve terminals (n). The basal membrane is shown by arrowheads. Scale bar $5 \mu \mathrm{m}$. 
$1 \mathrm{C}$ shows the electron micrograph of two MCs in the touch dome which had beforehand physiologically shown SAI responses to mechanical stimulation. The shape of these two Merkel cells (M) was that of dendritic-shaped Merkel cells rather than that of roundish-shaped Merkel cells. These Merkel cells were also characterized as having irregular contours with thick cytoplasmic processes (asterisks). The cytoplasmic process including abundant neurosecretory granules spread to the basal membrane (arrowheads) and faced toward the basal membrane. This characteristic feature resembles the DMCs as reported by Tachibana et al. (7). On the other hand, a few neurosecretory granules were situated in the cytoplasm between the neucleus of the dendriticshaped Merkel cells and associated nerve terminals (Fig. IC). Most of dendritic-shaped Merkel cells in the touch domes were in contact with nerve terminals (n) and hardly any DMCs without contacts to nerve terminals could be observed in the touch domes. If DMCs as described in Tachibana et al. (7) existed in the epithelial rete ridge of the touch domes, they would be quite rare. In the ultrastructural observations, we concluded that dendritic-shaped Merkel cells in hamster touch domes were intermediate between features of the RMC and DMC in the rodent palatine mucosa (7), since most dendritic-shaped Merkel cells contacted nerve terminals.

The function of Merkel cells is not yet clear, and the following functions are theorized: 1) transducer of SAI mechanoreceptor $(2,3,9) ; 2)$ target cell of growing nerve elements $(6,12) ; 3)$ trophic action of the neural elements and keratinocyte differentiation $(6,12)$, etc. It has been also proposed that polymorphism of Merkel cells may imply a functional heterogenetity of the cells (7). However, we concluded that the function of the roundish-shaped Merkel cells and dendriticshaped Merkel cells in the hamster touch dome are mechano-electric transducers of SAI mechanoreceptor since most of roundish-shaped Merkel cells and dendritic-shaped Merkel cells contacted nerve terminals and possessed stretch activated ion channels (11). It is hard to surmise the function of Merkel cells, because the shape of Merkel cells differs only on the light microscopic observation level. Investigation of the function of DMCs as described by Tachibana et al. (7) is required.

\section{Acknowledgments}

The authors wish to thank Miss Miwako Suzuki for her technical. This study was made possible by a generous grant from the Oral Health Science Center, 962B02 and 992C01, at Tokyo Dental College.

\section{REFERENCES}

1. Calderon-Solt L. and Solt D. B. (1984) Touch domes and Merkel cells in hamster cheek pouch epithelium. Archs. oral Biol. 29, 569-573.

2. Chan E., Yung W. -H. and Baumann K. I. (1996) Cytoplasmic $\mathrm{Ca}^{2+}$ concentrations in intact Merkel cells of an isolated, functioning rat sinus hair preparation. Exp. Brain Res. 108, 357-366.

3. Iggo A. and Muir A. R. (1969) The structure and function of a slowly adapting touch corpuscle in hairy skin. $J$. Physiol. (Lond.) 200, 763-796.

4. Kim D. -K. and Holbrook K. A. (1995) The appearance, density, and distribution of Merkel cells in human embryonic and fetal skin: Their relation to sweat gland and hair follicle development. J. Invest. Dermatol. 104, 411-416.

5. Moll R., Moll I. and Franke W. W. (1984) Identification of Merkel cells in human skin by specific cytokeratin antibodies: Changes of cell density and distribution in fetal and adult plantar epidermis. Differentiation 28, 136154.

6. Tachibana T. (1995) The Merkel cell: Recent findings and unresolved problems. Arch. Histol. Cytol. 58, 379-396.

7. Tachibana T., Yamamoto H., Takehashi N., Kamegai T., Shibanai S., Iseki H., Nawa T. (1997) Polymorphism of Merkel cells in the rodent palatine mucosa: Immunohistochemical and ultrastructural studies. Arch. Histol. Cytol. 60, 379-389.

8. Tazaki M. and Sakada S. (1994) Merkel cell-neurite complexes in touch domes of hamster cheek pouch. Bull. Tokyo Dent. Coll. 35, 95-98.

9. Tazaki M. and Iggo A. (1995) SAI-type mechanoreceptors in the buccal pouch of the golden hamster (Mesocricetus auratus). Prim. Sensory Neuron 1, 157-169.

10. Tazaki M. and Suzuki T. (1998) The study of voltagedependent $\mathrm{Cat}^{2+}$ channels in single Merkel cells in hamster cheek pouch mucosa. Dentistry in Japan 34, 28-31.

11. Tazaki M. and Suzuki T. (1998) Calcium inflow of hamster Merkel cells in response to hyposmotic stimulation indicate a stretch activated ion channel. Neurosci. Lett. 243, 69-72.

12. Vos P., Stark F. and Pittman R. N. (1991) Merkel cells in vitro: Production of nerve growth factor and selective interaction with sensory neurons. Dev. Biol. 144, 28I-300. 